\title{
Mini-Spin y Mini-Spin-VR: equivalencia entre test de ansiedad virtuales y tradicionales
}

\section{(Mini-Spin and Mini-Spin-VR: equivalence between virtual and traditional anxiety test)}

\begin{abstract}
Verónica Egas-Reyes ${ }^{1}$, Raúl Hinojosa-Alcocer², Diego Ordóñez-Camacho²
Resumen:

Las tecnologías de realidad virtual permiten representar casi cualquier ambiente, real o ficticio, en una escena virtual. Estas escenas facilitan que un usuario pueda integrarse dentro de un laboratorio controlado y participar en diferentes estudios científicos. En muchos campos del quehacer científico, como por ejemplo la psicología, se han integrado ya estas tecnologías, sin embargo, el área todavía presenta muchos problemas abiertos. Este trabajo busca conocer si los cada vez más depurados ambientes virtuales pueden ser capaces de remplazar a las pruebas psicológicas realizadas de manera tradicional. Esto es lo que en esta investigación se planteó, al recrear virtualmente tres situaciones humanas y vincularlas con un test de ansiedad tradicional. Mediante el doble test unilateral de equivalencia, los resultados mostraron una equivalencia estadística significativa.
\end{abstract}

Palabras clave: TIC; realidad virtual, tecnología aplicada a la psicología; test psicológico; mini-spin.

\begin{abstract}
:
Virtual reality technologies enable the representation of a vast array of existing and fictitious environments into virtual, digital scenes. These scenes allow for the easy integration of users into a controlled laboratory, allowing them to participate on a variety of scientific studies. Many different areas, like psychology, have already integrated virtual technologies into its field; nevertheless, there are still many open problems related. The main focus of this work is to find out if the virtual environments could replace traditional pen and paper psychological test. Thanks to the use of the two one sided equivalence test, results showed significant statistical equivalence.
\end{abstract}

Keywords: ICT; virtual reality; technology applied to psychology; psychological test; mini-spin.

\section{Introducción}

La realidad virtual es una tecnología que, mediante sonidos, imágenes y texturas, permite tomar algún aspecto del mundo cotidiano y simularlo digitalmente, transformándolo en un ambiente virtual. Más aún, no es solo el mundo concreto el cual puede ser sujeto de este proceso, sino que es posible crear nuevos mundos, mundos fantasiosos o imaginarios, con sus propias leyes físicas y regulaciones.

Estos ambientes virtuales permiten que el usuario interactúe con ellos de manera similar al mundo real, mediante sentidos como la vista, el oído o inclusive el tacto. Al recibir estos estímulos multidimensionales, el usuario ejecuta ciertas acciones, dentro de los límites del entorno tecnológico, y obtiene una retroalimentación digital inmediata que permite la interacción como si de la realidad se tratara.

\footnotetext{
1 Pontificia Universidad Católica del Ecuador, Quito - Ecuador ( megas776@puce.edu.ec ).

2 Universidad Tecnológica Equinoccial, Quito - Ecuador ( \{harf86320, dordonez\} @ute.edu.ec ).
} 
Las posibilidades digitales que se abren son innumerables dado que la tecnología permite crear escenarios inmersivos ultrarealistas en los cuales el usuario puede integrarse dentro de un laboratorio controlado y participar en diferentes estudios científicos (Earnshaw, 2014).

Actualmente la tecnología está íntimamente relacionada con muchos campos del quehacer humano, como la adaptación al contexto y entorno (Loayza, Proaño, \& Ordóñez, 2013). En lo psicológico también se encuentran aportes tecnológicos, especialmente en la psicología educativa, con versiones digitales y adaptaciones de pruebas de aprendizaje, psicoeducativas y cognitivas. Sin embargo, en el campo de la psicología clínica, en temas de trastornos clínicos o relaciones afectivas-emocionales aún hay mucho por trabajar. Las pruebas o baterías de test clínicos, por ejemplo, se realizan en su mayoría con papel y lápiz, sin apoyo de la tecnología. Existen, a pesar de todo, varios trabajos que buscan incorporarla y, más específicamente la realidad virtual, al campo de la psicología clínica.

Es justamente sobre este tópico que gira en la actualidad una de las grandes discusiones: conocer si los cada vez más depurados ambientes virtuales pueden ser capaces de remplazar a las pruebas psicológicas realizadas de manera tradicional. Esto es lo que en esta investigación se planteó, al recrear virtualmente tres situaciones humanas y vincularlas con un test de ansiedad, para así continuar con el debate previamente abierto (Hinojosa-Alcocer, Ordóñez-Camacho, \& Egas-Reyes, 2017) sobre su equivalencia.

\subsection{Trabajos relacionados}

Varias áreas relacionadas con la salud y la psicología han recibido ya el aporte de la realidad virtual. En el campo de la medicina, por ejemplo, Schlickum et al. (2009) emplearon en primer lugar el entrenamiento en videojuegos con miras a mejorar luego el rendimiento en simuladores para cirugía endoscópica construidos en ambientes virtuales. Se mostró en el estudio que un entrenamiento sistemático con videojuegos, aumenta la similitud contextual con la simulación quirúrgica.

Ya en el área del tratamiento de pacientes con necesidad de soporte, se encuentran casos como el de You et al. (2005), quienes utilizaron la realidad virtual para tratar pacientes con problemas cerebrovasculares. En este estudio se prepararon ambientes virtuales caracterizados por escenas interactivas de la vida real. El objetivo de estas escenas era enseñar de nuevo los movimientos básicos al sistema motor del cerebro.

Más integrado al campo de la psicología y a los desórdenes que esta estudia, North et al. (2015) trabajaron con la realidad virtual para tratar pacientes con problemas para hablar en público. Luego de cinco semanas de tratarse con el simulador virtual, los pacientes presentaron una mejoría significativa.

Rothbaum et al. (2001) trataron a pacientes con desórdenes de estrés postraumático, veteranos vietnamitas, exponiéndoles de forma repetida a dos escenas virtuales que recreaban ambientes vietnamitas relacionados con la guerra. Se reportó que los síntomas de estrés postraumático en los pacientes, se redujeron entre un $15 \%$ y un $67 \%$.

Uno de los estudios emblemáticos en el campo de la psicología clínica fue realizado por Diemer et al. (2015), quienes analizaron fobias de tipo animal (arañas) y comprobaron que la reacción de miedo ante el aparecimiento del animal se observa en un ambiente virtual con similar intensidad que en un ambiente real.

Pedroli et al. (2017) utilizaron la realidad virtual como un método de rehabilitación con niños para quienes es difícil seguir un tratamiento más tradicional, dado que tienden a aburrirse fácilmente. Kandalaft et al. (2013), investigaron los beneficios que el uso de una plataforma de realidad virtual puede proporcionar a niños autistas, mejorando sus habilidades sociales y su cognición.

En el campo más específico de la psicometría, La Paglia et al. (2014) comparan los resultados obtenidos con una versión tradicional y otra virtual de la batería de test neuropsicológicos V-MET, encontrando que las dos están correlacionadas. De la misma manera, Shiban et al. (2016) comparan las versiones tradicional y virtual del TSST, 
encontrando que la realidad virtual podría servir como una herramienta estandarizada para la inducción de estrés en condiciones experimentales.

\subsection{Contribuciones}

Como se puede evidenciar, la creación de software para pruebas psicológicas clínicas está ya iniciando, pero su uso y acceso es todavía limitado. Este trabajo intenta dar su aporte para llenar la brecha, mediante las siguientes contribuciones:

- De manera general aportar al entendimiento y comprensión de los procesos y mecanismos mediante los cuales se puede tomar un test tradicional en el área de la psicología clínica y representarlo en un sistema de realidad virtual.

- Proporcionar elementos que permitan una verificación inicial, en un ambiente controlado, de la equivalencia estadística entre las modalidades tradicional y virtual del test psicológico.

- Ofrecer específicamente una demostración experimental tomando el caso del test sicológico Mini-Spin (Connor, Kobak, Churchill, Katzelnick, \& Davidson, 2001), el cual se utiliza para evaluar rápidamente la presencia e intensidad del desorden de ansiedad social.

\section{Metodología}

Para cumplir los objetivos del trabajo se planificaron dos etapas de primer nivel. Inicialmente se construyó un prototipo que escenificó tres ambientes virtuales; cada uno de estos ambientes representó una de las preguntas del Mini-Spin, que se pueden ver en la Tabla 1.

Tabla 1. Preguntas del cuestionario Mini-Spin

\begin{tabular}{cc}
\hline$\#$ & Pregunta \\
\hline 1 & El miedo a avergonzarme causa que evada hacer cosas o hablar con personas \\
2 & Evito actividades en las cuales soy el centro de atención \\
3 & Sentirme avergonzado o verme estúpido están entre mis peores miedos \\
\hline
\end{tabular}

En segundo lugar, para probar el prototipo, se trabajó con un grupo de participantes a quienes se aplicó el test psicológico en sus versiones tradicional y virtual. La información resultante se analizó estadísticamente en búsqueda de indicios significativos que el método virtual puede ser considerado equivalente al tradicional.

Para desarrollar el prototipo de realidad virtual se utilizó el motor gráfico Unity3D (Linowes, 2015), donde los componentes de código fueron diseñados para el control de flujo de cada escena, buscando tanto su congruencia, como controlar el input de usuario, el movimiento de la vista y las reacciones físicas entre objetos virtuales. Todos los objetos virtuales fueron maquetados, modelados y animados en el sistema de modelado Blender (Mullen, 2012) y fueron exportados al motor gráfico manteniendo su textura y detalle. Su funcionalidad original fue probada y se añadieron disparadores de sonido de acuerdo con cada actividad propuesta en los eventos virtuales generados para Mini-Spin. Se utilizó hardware especializado para realidad virtual, principalmente las gafas Oculus Rift (Donovan, 2017), las cuales permiten al usuario sentirse dentro de la escena (LaValle, Yershova, Katsev, \& Antonov, 2014).

Según el protocolo propio del Mini-Spin (Connor et al., 2001), el cuestionario se entregó en papel a los participantes, a quienes se les dio la consigna de responder basados en sus experiencias cercanas, preferentemente de la semana inmediatamente anterior. Los participantes respondieron indicando qué tan característica en ellos es la conducta descrita en cada pregunta; las respuestas se ponderaron conforme a los valores de la Tabla 2. 
El protocolo de uso del prototipo de realidad virtual consistió en indicar a los participantes que debían ponerse las gafas de realidad virtual y que posteriormente verían tres escenas diferentes, una a continuación de la otra. Se les indicó que al inicio de cada escena se presentarían instrucciones específicas de lo que deberían hacer dentro de la misma y que al final de la escena se les presentaría en el entorno virtual la pregunta "¿Qué tan avergonzado se sintió en esta experiencia?". Los participantes debían responder la pregunta seleccionando una de las alternativas, que son las mismas presentadas en la Tabla 2.

Tabla 2. Respuestas posibles y sus ponderaciones

\begin{tabular}{ll}
\hline Respuesta & Peso \\
\hline Para nada & 0 \\
Un poco & 1 \\
Algo & 2 \\
Mucho & 3 \\
Extremadamente & 4 \\
\hline
\end{tabular}

Para establecer la relación pregunta / evento-virtual, se seleccionó una muestra de 30 sujetos de prueba voluntarios, estudiantes universitarios de diversas carreras. Se les aplicó en primer lugar el test Mini-Spin tradicional, y luego de una breve espera, se les pidió utilizar el prototipo de realidad virtual. Finalmente, la equivalencia estadística de comparación entre los dos métodos se realizó mediante el uso de la prueba de equivalencia doble unilateral, conocida en inglés como Two One-Sided Test (TOST) (Dong, Tsong, \& Shen, 2014), mediante $\mathrm{R}$ (Ihaka \& Gentleman, 1996), y el paquete estadístico de equivalencia (Robinson, 2016); se aplicaron los parámetros: épsilon 1, alfa 0.05 y emparejamiento verdadero.

\section{Resultados}

\subsection{Virtualización de las preguntas del Mini-Spin en escenas}

Elegir un método o técnica para trasladar el contexto de una pregunta hacia un evento virtual es complejo, al existir demasiadas variables involucradas en la forma de ver una pregunta y su representación en la realidad. El simple hecho de elegir el lugar de desarrollo puede variar en numerosas combinaciones e impactar en la comparación entre un texto y un ambiente virtual.

Para la investigación se propusieron tres escenas, cada una de ellas ligadas a una de las preguntas del Mini-Spin, enfocándose en ciertos elementos emocionales específicos. La primera escena se dirige a la generación de vergüenza, timidez y retraimiento relacionados directamente con el cuerpo, la imagen corporal y la relación con lo social. La segunda escena trata la exposición del sujeto a un público desconocido y apunta a la generación de sensaciones de falla, rechazo y angustia. Finalmente, la tercera escena, aborda la exposición del sujeto a un público conocido (sus pares) y se direcciona a la vergüenza, bochorno y retraimiento. Todas estas sensaciones y emociones que pueden surgir en el momento de la prueba están directamente relacionadas con la ansiedad.

\subsubsection{Primera pregunta: el miedo a avergonzarme causa que evada hacer cosas o hablar con personas}

Existen muchas escenas que pudieron satisfacer esta exclamación; sin embargo, se generó la escena de la Figura 1, en la cual el sujeto de prueba se encuentra a punto de ingresar a una fiesta de piscina. Esta posición inicial del jugador se justifica ya que en todas las escenas menos en la última el sujeto de prueba inicia en un lugar aislado, fuera aún de 
toda la interacción con los personajes, con el fin de mostrarle primero la información general de qué ocurre en cada escena y que no se enfoque aun en los detalles que deberían hacerle sentir cohibido y rodeado de gente.

Después de saber en qué situación se encuentra el sujeto de prueba se le provee de instrucciones claras de cuál debe ser su papel en la experiencia, así como la forma de utilizar los controles para poder caminar, tomar objetos etc.

Finalmente, el sujeto se encuentra dentro de la fiesta de piscina donde encontramos muchos objetos acorde con la temática como sillas, mesas, objetos humanoides y un sol radiante; estos objetos ayudan a que el usuario relacione aún más en donde se encuentra. Se tuvo en cuenta una piscina ya que la vestimenta que debe utilizarse puede conllevar a sentir un poco de ansiedad si se encuentra rodeado de gente por lo cual se eligió un cuerpo en bañador para la cámara del jugador.

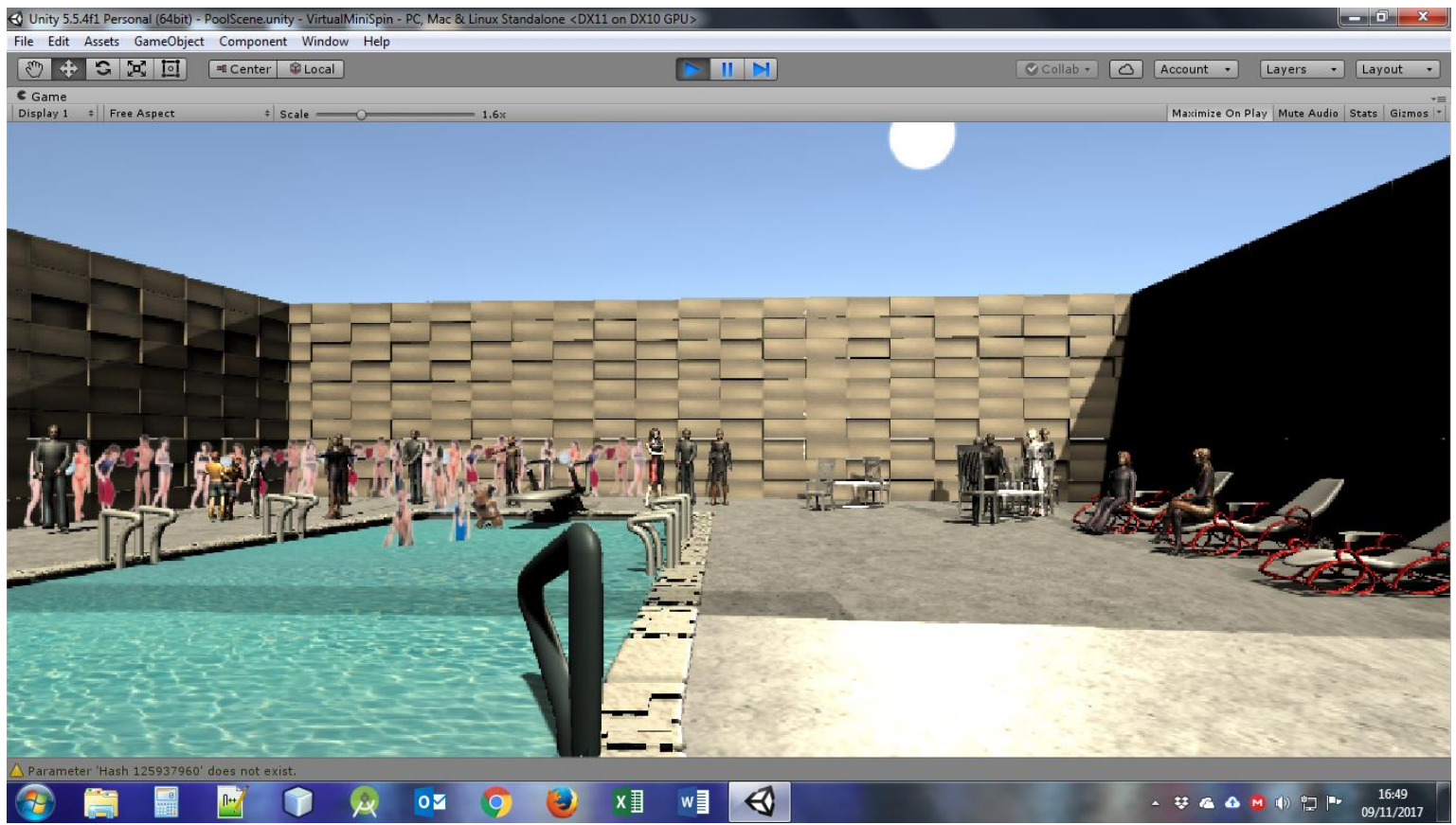

Figura 1. Escena de la aplicación relacionada con la primera pregunta

\subsubsection{Segunda pregunta: evito actividades en las cuales soy el centro de atención}

Se generó la escena virtual de la Figura 2, en la cual el sujeto inicia con una introducción clara de qué está sucediendo en la nueva experiencia y cuál debería ser su primer paso para iniciarla, citando el texto del panel informativo: "en esta experiencia te encuentras en un estadio a punto de dar un discurso frente a todas estas personas. Camina hacia el recuadro amarillo". Luego observamos el público en el graderío con una leve animación en grupo para dar la sensación de público en masa, los modelos de esta escena son más básicos en tema de diseño y texturas ya que al ser muchos el procesamiento gráfico y de cpu deberían ser mayores para evitar latencia y rendimiento pobre de la experiencia.

Se muestran nuevas indicaciones para iniciar el discurso que dura poco tiempo; al ingresar a la plataforma de instrucciones inicia un sonido de aplauso cuya fuente fue tomada de una reunión social de grado en una universidad del Ecuador. Al terminar el discurso se emite otro disparador de sonido el cual son abucheos por parte del público y al terminar este disparador se muestra el cuadro final de calificación que llevará a nuestra siguiente experiencia. 


\subsubsection{Tercera pregunta: sentirme avergonzado o verme estúpido están entre mis peores miedos}

La última escena está basada en una experiencia muy común en el ámbito de la educación. El sujeto de prueba se encuentra en la parte final de una clase junto a un maestro y tres estudiantes más; la profesora nota que el sujeto no está prestando atención por lo que se activa un audio autoritario y señala con el dedo al estudiante para formularle la pregunta. Luego los estudiantes regresan a observar al sujeto causando la posible reacción de intimidación y de vergüenza.

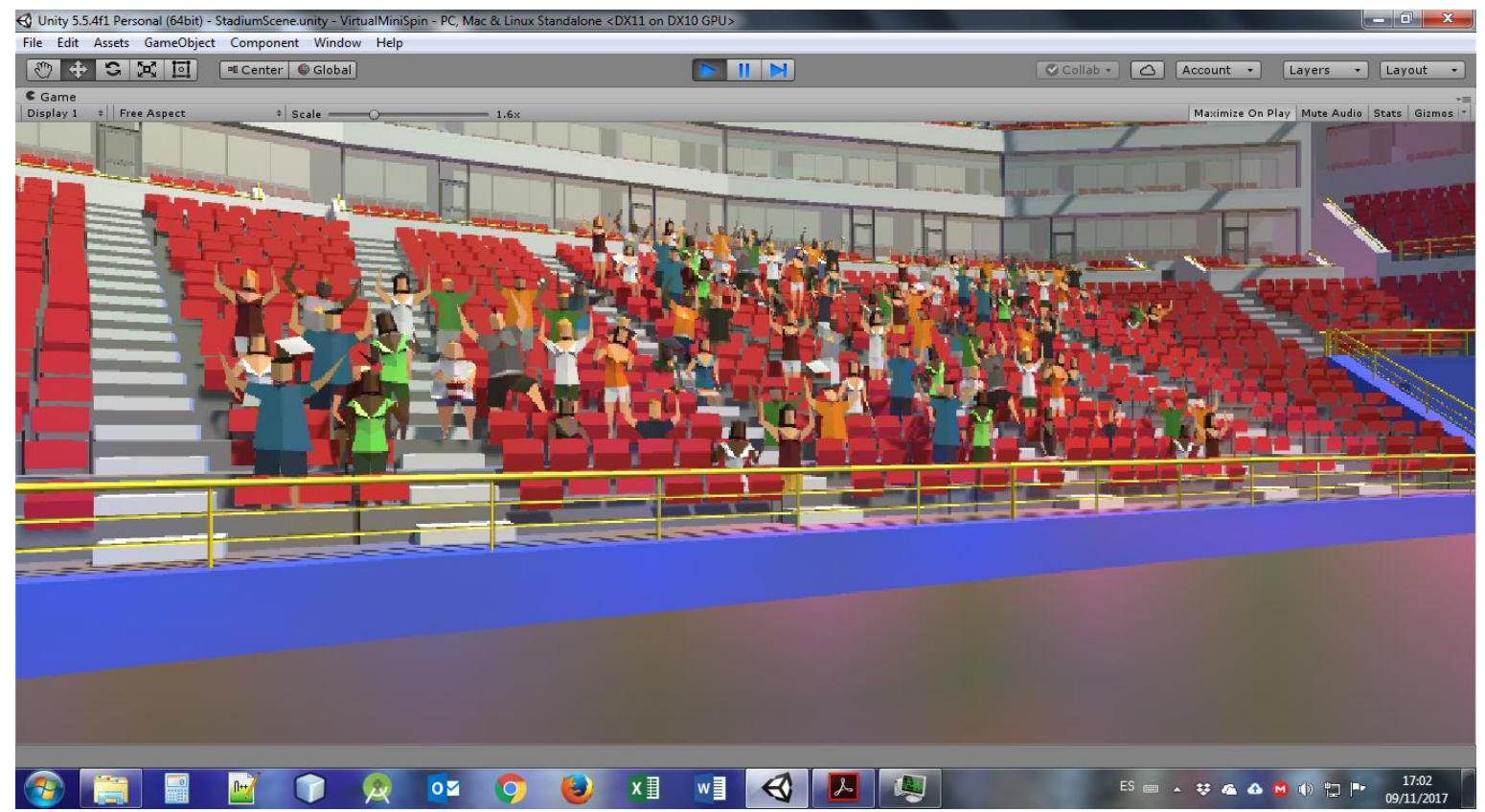

Figura 2. Escena de la aplicación relacionada con la segunda pregunta

En la Figura 3 se presenta el momento en el cual todos los estudiantes regresan a ver al sujeto de prueba al no contestar la pregunta.

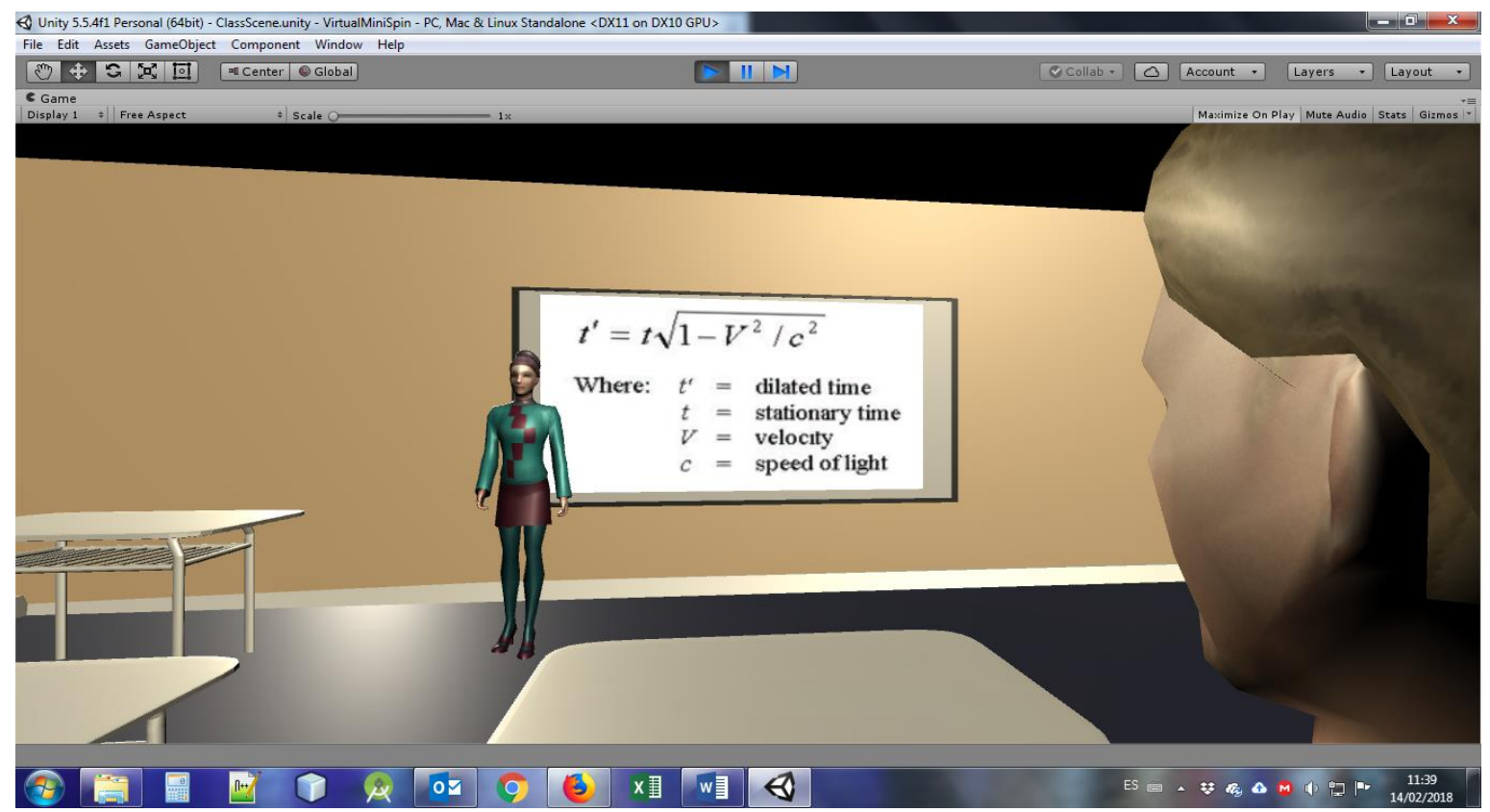

Figura 3. Escena de la aplicación relacionada con la tercera pregunta 


\subsection{Estadísticos y comparativos}

La ansiedad social en Mini-Spin es medida por las respuestas otorgadas por el sujeto, después de estar expuesto a los ambientes virtuales. Como en el estudio de Diemer et al. (2015) sobre la aracnofobia, mientras más se involucren los sentidos, la credibilidad del ambiente virtual será mayor.

En la Figura 4 vemos que la comparación en la pregunta 1 referente a ambos test obtuvo resultados muy diferentes; cabe tomar en cuenta que para las opciones 3 (mucho) y 4 (extremadamente), a lo largo de todas las pruebas los niveles siempre fueron bajos. En la opción 0 (para nada) observamos una diferencia de 16 a 4 y en respuestas de 1 (un poco) ocurre lo contrario, de 8 a 20.

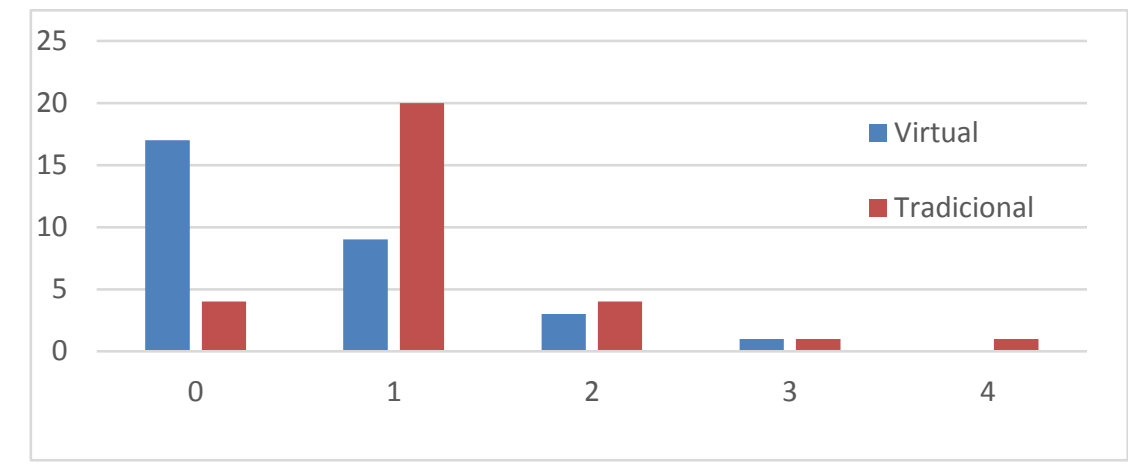

Figura 4. Comparación de respuestas: virtual/tradicional en la pregunta 1

En la pregunta 2 la diferencia entre respuestas se ajusta un poco con resultados congruentes en la respuesta 1 (un poco) y vuelve a existir una diferencia significativa en 0 (para nada) con un nivel mayor en la escena dos, como muestran los resultados de la Figura 5.

En la última escena del prototipo los resultados de la Figura 6 son mucho más correlacionados al no existir una diferencia tan significativa en respuestas generales. Podría deberse a que en esta escena se logró un ambiente mucho más personal, presentado al sujeto de prueba con más detalle y acciones de emoción,

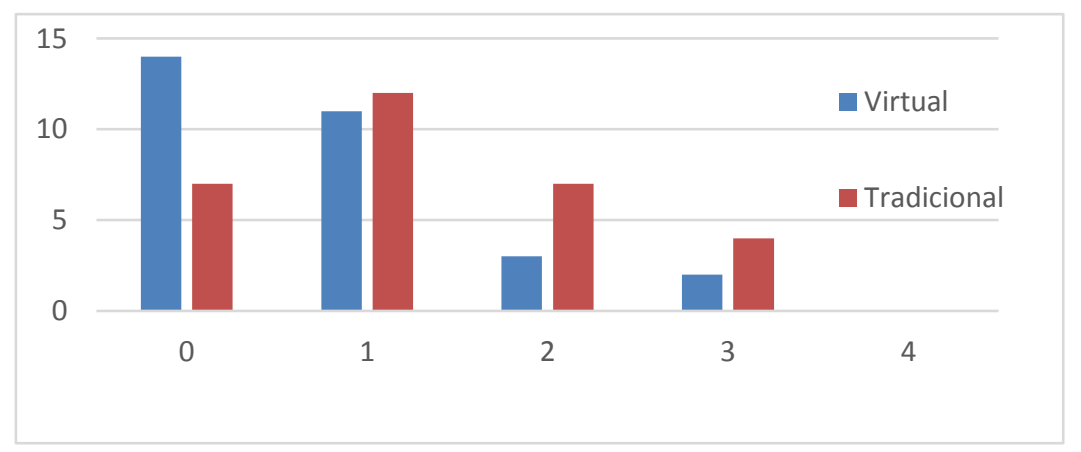

Figura 5. Comparación de respuestas: virtual/tradicional en la pregunta 2 


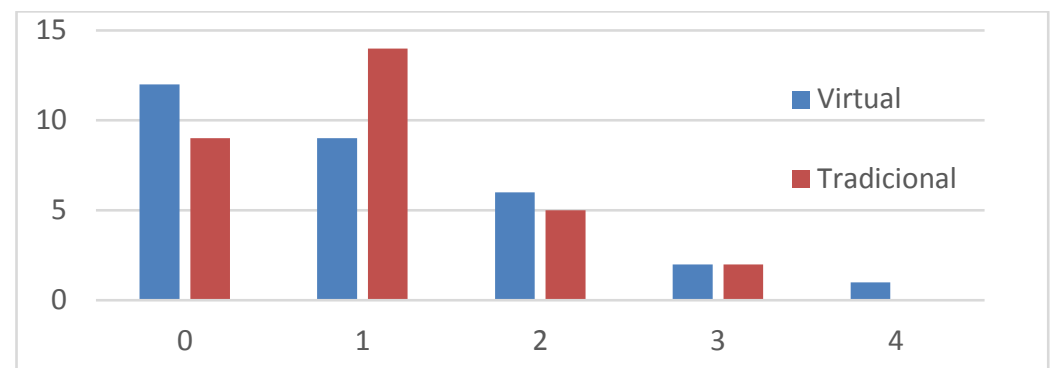

Figura 6. Comparación de respuestas: virtual/tradicional en la pregunta 3

En la Tabla 3 se presenta la información general de los resultados al aplicar la prueba de equivalencia estadística TOST a las tres preguntas; se comparan los resultados de las versiones virtual y tradicional.

Tabla 3. Resultados de TOST sobre preguntas virtual/tradicional

\begin{tabular}{lrrr}
\hline & Pregunta 1 & Pregunta 2 & Pregunta 3 \\
\hline Media Virtual & 0.600 & 0.767 & 1.033 \\
Media Tradicional & 1.167 & 1.267 & 1.000 \\
Varianza Virtual & 0.662 & 0.806 & 1.206 \\
Varianza Tradicional & 0.695 & 0.961 & 0.759 \\
Media de las diferencias & -0.567 & -0.500 & 0.033 \\
Límite inferior TOST & -0.889 & -0.905 & -0.369 \\
Límite superior TOST & -0.244 & -0.095 & 0.436 \\
Valor-p TOST & 0.015 & 0.022 & 0.001 \\
\hline épsilon =1 & & & \\
alfa $=0.5$ & & & \\
\hline
\end{tabular}

\section{Discusión}

Existen ventajas visibles cuando utilizamos ambientes virtuales como herramienta de investigación en la psicología, como destacan Blascovich et al. (2002), al crear situaciones controladas en un ambiente artificial, habilitando un control preciso en la entrega del estímulo; sin embargo, desventajas también pueden encontrarse como crear estímulos complejos que requieren de alta exactitud que pueden provocar imperfecciones en los modelos renderizados, limitaciones del visor y lentitud significante entre el sensor de movimiento y el visor tridimensional. Limitaciones de procesamiento gráfico en computadores o las gafas de realidad virtual incluso hoy en día pueden ser la causa que la experiencia no sea tan creíble lo cual podría ser un factor que afecte los resultados.

Para el análisis de equivalencia estadística se optó por una prueba de equivalencia probabilística sobre una distribución $T$ tradicional, ya que las distribuciones $T$ especialmente la T de Student buscan encontrar una diferencia significativa entre colas de datos, mientras que una prueba de equivalencia difiere, al no obtener interpretaciones erradas sobre la probabilidad $p$ de los resultados como Lakens denota: "TOST rechaza efectos suficientemente grandes para ser considerados como información valiosa. La adopción de pruebas de equivalencia evitará las malas interpretaciones comunes de valores p no significativos" (Lakens, 2017).

En relación con el análisis estadístico TOST podemos ver que para todas las preguntas el valor $p$ es inferior al alfa de 0.05 lo cual permite rechazar la hipótesis nula de diferencia estadística; adicionalmente los límites superior e inferior del intervalo de equivalencia caen dentro de la región de similitud épsilon \pm 1 .

Empíricamente, encontrar estas determinantes en la ansiedad social es un problema más fácil ya que "como los miedos sociales se comportan generalmente, tienden a ser más cognoscitivos en la naturaleza que una fobia en específico" (Schuemie, van der Straaten, Krijn, \& van der Mast, 2001), es decir que la ansiedad social debe ser vista o estar frente a 
ella para saber cómo el sujeto va a reaccionar lo cual justifica el proceso y la elección de la tecnología de este proyecto.

\section{Conclusiones y recomendaciones}

Realidad virtual y psicología tienen una relación y se ligan de manera efectiva tanto al enfrentar procesos de diagnóstico, como al involucrarse en el tratamiento de una diversidad de problemáticas psicológicas. Cabe recalcar, sin embargo, que existe un sinnúmero de problemáticas y síntomas, dentro de los confines de la Psicología Clínica, que requieren de manera absoluta un contacto humano directo a todo nivel, incluido el momento de la toma de la prueba (psicólogo evaluador y paciente). Esto es fundamental para que se fortalezca la relación, lo cual influenciará de manera directa a lo largo de todo el trabajo psicológico. Sin embargo, existen otras áreas o situaciones en las cuales sería posible el articular estos dos campos de conocimiento, como por ejemplo, con pruebas como la expuesta en este trabajo.

La equivalencia estadística encontrada al aplicar la prueba en sus dos modalidades, tradicional y virtual, fue satisfactoria y significativa. De manera especial esto se demostró en aquellas escenas que por su naturaleza lograron involucrar, por un lado un nivel más alto de detalle, y por otro, acciones que pudieron generar distintos tipos de emociones en el sujeto. Este detalle, sin embargo, se enfrenta con ciertos límites tecnológicos dados tanto por el poder de procesamiento computacional requerido en el renderizado y la presentación de las escenas, como por el esfuerzo hora/hombre exigido en la etapa de diseño.

El trabajo a futuro que esta línea de investigación abre, se centra especialmente en dos direcciones. Primero, se debe ampliar el espectro de test psicológicos clínicos en los cuales pueda intervenir exitosamente la tecnología de realidad virtual. Luego, directamente relacionado con el trabajo realizado en este proyecto, es pertinente ahondar en el estudio de representaciones alternativas de escenas que puedan tener mayor y mejor impacto en distintas poblaciones y entornos.

\section{Agradecimientos}

La colaboración interinstitucional requerida para esta investigación fue posible gracias al Proyecto de Investigación para el Desarrollo (PRD-ARES/CCD) Bélgica-Ecuador "Renforcement des espaces de médiation communautaire des jeunes dans les situations de violence et de souffrance psychosociales".

\section{Bibliografía}

Blascovich, J., Loomis, J., Beall, A. C., Swinth, K. R., Hoyt, C. L., \& Bailenson, J. N. (2002). Immersive Virtual Environment Technology as a Methodological Tool for Social Psychology. Psychological Inquiry, 13(2), 103-124. https://doi.org/10.1207/S15327965PLI1302_01

Connor, K. M., Kobak, K. A., Churchill, L. E., Katzelnick, D., \& Davidson, J. R. T. (2001). Mini-SPIN: A brief screening assessment for generalized social anxiety disorder. Depression and Anxiety, 14(2), 137-140. https://doi.org/10.1002/da.1055

Diemer, J., Alpers, G. W., Peperkorn, H. M., Shiban, Y., \& Mühlberger, A. (2015). The impact of perception and presence on emotional reactions: a review of research in virtual reality. Frontiers in Psychology, 6. https://doi.org/10.3389/fpsyg.2015.00026

Dong, X., Tsong, Y., \& Shen, M. (2014). Equivalence Tests for Interchangeability Based on Two One-Sided Probabilities. Journal of Biopharmaceutical Statistics, 24(6), 13321348. https://doi.org/10.1080/10543406.2014.941987

Donovan, J. (2017). Mastering Oculus Rift Development. Packt Publishing Ltd.

Earnshaw, R. A. (2014). Virtual Reality Systems. Academic Press. 
Hinojosa-Alcocer, R., Ordóñez-Camacho, D., \& Egas-Reyes, V. (2017). Virtual Reality Applied to Psychological Anxiety Tests (Vol. 2). Presentado en International Conference on Information Systems and Computer Science.

Ihaka, R., \& Gentleman, R. (1996). R: A Language for Data Analysis and Graphics. Journal of Computational and Graphical Statistics, 5(3), 299-314. https://doi.org/10.1080/10618600.1996.10474713

Kandalaft, M. R., Didehbani, N., Krawczyk, D. C., Allen, T. T., \& Chapman, S. B. (2013). Virtual reality social cognition training for young adults with high-functioning autism. Journal of autism and developmental disorders, 43(1), 34-44.

La Paglia, F., La Cascia, C., Cipresso, P., Rizzo, R., Francomano, A., Riva, G., \& La Barbera, D. (2014). Psychometric assessment using classic neuropsychological and virtual reality based test: A study in obsessive-compulsive disorder (OCD) and schizophrenic patients. En International Symposium on Pervasive Computing Paradigms for Mental Health (pp. 23-32). Springer.

Lakens, D. (2017). Equivalence Tests: A Practical Primer for t Tests, Correlations, and Meta-Analyses. Social Psychological and Personality Science, 8(4), 355-362. https://doi.org/10.1177/1948550617697177

LaValle, S. M., Yershova, A., Katsev, M., \& Antonov, M. (2014). Head tracking for the Oculus Rift. En 2014 IEEE International Conference on Robotics and Automation (ICRA) (pp. 187-194). https://doi.org/10.1109/ICRA.2014.6906608

Linowes, J. (2015). Unity Virtual Reality Projects. Birmingham Mumbai: Packt Publishing ebooks Account.

Loayza, A., Proaño, R., \& Camacho, D. O. (2013). Aplicaciones sensibles al contexto. Tendencias actuales. Enfoque UTE, 4(2), 95-110. https://doi.org/10.29019/enfoqueute.v4n2.31

M. North, M., M. North, S., \& R. Coble, J. (2015). VIRTUAL REALITY THERAPY: AN EFFECTIVE TREATMENT FOR THE FEAR OF PUBLIC SPEAKING. International Journal of Virtual Reality (IJVR), 03(3), 1-6.

Mullen, T. (2012). Mastering Blender. John Wiley \& Sons.

Pedroli, E., Padula, P., Guala, A., Meardi, M. T., Riva, G., \& Albani, G. (2017). A Psychometric Tool for a Virtual Reality Rehabilitation Approach for Dyslexia. Computational and mathematical methods in medicine, 2017.

Robinson, A. (2016). equivalence: Provides Tests and Graphics for Assessing Tests of Equivalence (Versión 0.7.2). Recuperado a partir de https://cran.rproject.org/web/packages/equivalence/index.html

Rothbaum, B. O., Hodges, L. F., Ready, D., Graap, K., \& Alarcon, R. D. (2001). Virtual reality exposure therapy for Vietnam veterans with posttraumatic stress disorder. The Journal of clinical psychiatry.

Schlickum, M. K., Hedman, L., Enochsson, L., Kjellin, A., \& Felländer-Tsai, L. (2009). Systematic Video Game Training in Surgical Novices Improves Performance in Virtual Reality Endoscopic Surgical Simulators: A Prospective Randomized Study. World Journal of Surgery, 33(11), 2360. https://doi.org/10.1007/s00268-009-0151-y

Schuemie, M. J., van der Straaten, P., Krijn, M., \& van der Mast, C. A. P. G. (2001). Research on Presence in Virtual Reality: A Survey. CyberPsychology \& Behavior, 4(2), 183-201. https://doi.org/10.1089/109493101300117884

Shiban, Y., Diemer, J., Brandl, S., Zack, R., Mühlberger, A., \& Wüst, S. (2016). Trier Social Stress Test in vivo and in virtual reality: Dissociation of response domains. International Journal of Psychophysiology, 110(Supplement C), 47-55. https://doi.org/10.1016/j.ijpsycho.2016.10.008

You, S. H., Jang, S. H., Kim, Y.-H., Hallett, M., Ahn, S. H., Kwon, Y.-H., Lee, M. Y. (2005). Virtual Reality-Induced Cortical Reorganization and Associated Locomotor Recovery in Chronic Stroke. Stroke, 36(6), 1166-1171. https://doi.org/10.1161/01.STR.0000162715.43417.91 